\title{
Exploring the Effects of Mental Imagery in the Solution Focused Approach
}

\author{
Masahiro Kawahara \\ Faculty of Humanities and Social Science, Iwate University, Iwate, Japan \\ Email:kawamasa@iwate-u.ac.jp
}

How to cite this paper: Kawahara, M. (2021) Exploring the Effects of Mental Imagery in the Solution Focused Approach. Open Journal of Medical Psychology, 10, 36-46.

https://doi.org/10.4236/ojmp.2021.102004

Received: February 19, 2021

Accepted: April 6, 2021

Published: April 9, 2021

Copyright $\odot 2021$ by author(s) and Scientific Research Publishing Inc. This work is licensed under the Creative Commons Attribution International License (CC BY 4.0).

http://creativecommons.org/licenses/by/4.0/

(c) (i) Open Access

\begin{abstract}
This study examined the effects of mental imagery in the solution-focused approach by evaluating the impact of positive self-image about the future on emotional states using the time machine question (which is a questioning technique used in the solution-focused approach). We compared the change in the emotional state of 270 participants, using the Japanese version of the Positive and Negative Affect Schedule (PANAS), before and after the intervention. The intervention conditions included: verbal description of one's positive future on a worksheet (the language description condition), and imagining one's positive future (the imagery condition). The results of the experiment showed that after the intervention, the scores of the imagery group on the positive and negative affect scales of the PANAS were significantly higher and lower, respectively, than those of the language description group. We also found that the amount of change in the scores of the positive and negative affect scales of the PANAS was significantly larger in the imagery group as compared to the language description group. These results indicate that interventions involving the imagining of one's future via the time machine question of the solution-focused approach have a more direct impact on emotional states than interventions using a language description. This suggests that mental imagery plays an important role in interventions carried out within the framework of the solution-focused approach.
\end{abstract}

\section{Keywords}

Mental Imagery, Solution-Focused Approach, Emotion State,

Time Machine Question

\section{Introduction}

The solution-focused approach (SFA), a type of brief therapy, is a counseling 
method created by Berg and Shazer at the Brief Family Therapy Center (BFTC) in America in the 1980s [1]. Recently, the SFA has become popular among medical, welfare, and education professionals around the world [2]. The SFA is characterized by focusing on solutions or the future rather than on the client's past problems. The SFA leads the client to find a solution to their problems or build a better future by focusing on the client's resources and strengths.

Questions such as the "miracle question", "time machine question", and "question to look for exceptions" have been developed within the research domain of SFA for use during client interviews to help the client imagine a positive situation. During an SFA interview, the therapist uses these questions to guide the client in problem-solving or imagining future positive situations. The client reflects upon the therapist's question and imagines a situation that is different from the present. Then the client talks to the therapist about problem-solving and positive self-future situations, as imagined by the therapist's questions. The client is expected to be able to imagine a vivid situation that helps in problem-solving or is a reflection of the desired positive future through this clienttherapist interaction. Mental images allow us to simulate the "pre-experience" of possible events [3] [4]. Therefore, the interaction of imagery between the therapist and the client, beginning with the question put forth by the therapist in the SFA interview, causes the client to create a simulated experience of how to solve their problems or achieve a better future. The client is expected to obtain a new awareness of problem-solving and a better future through this process.

The efficacy of mental images in the area of SFA has been indicated through case studies. For example, Berg and de Jong [5] argued that the therapist can share an image with the client through a discussion on what the client imagines as a problem-solving situation. They also reported the process by which the content of the talk about the image of the solution during the interview can lead to a shift in the therapy sessions from the cause of the problem to a way for problem-solving. Ng, Parikh, and Guo [6] reported an SFA interview using guided imagery for a Chinese female student suffering from emotional disorders due to the experience of losing her boyfriend, and showed that the symptoms of her illness improved after three interviews. In addition, recently, SFA interviews using guided imagery have gained traction in the field of sports psychology, and have been conducted for a golf player suffering from symptoms of the yips. In this case, SFA was found to be successful in reducing the severity of the player's yips during the round [7].

In addition to these case studies, quantitative studies have also reported that mental images play an important role in the efficacy of SFA. For example, Sklare, Sabella, and Petrosko [8] developed Solution-Focused Guided Imagery (SFGI) that combined guided imagery with solution-focused brief counseling. They then conducted interventions using SFGI with participants in skill-up seminars for professionals in the field of psychology. Bell et al. [9] [10] conducted a semi-structured interview based on SFGI for golf players suffering from symptoms of the yips. The results indicated that the number of yips during the round 
decreased for golfers who received the SFGI program after the intervention.

Considering the results and findings obtained from these previous studies, mental imagery is assumed to be an important factor related to the effectiveness of SFA interventions. However, there are overwhelmingly few experimental studies on mental images in the area of SFA. Hence, little is known about the efficacy of interventions using image guidance or the role of mental imagery in SFA intervention.

Recently, it has been found that mental imagery has a strong influence on emotions and motivation for behavior. In particular, many studies have been conducted in the research field of cognitive-behavioral therapy to assess the effect of image-based interventions on emotional disorders such as depression and anxiety disorders [4] [11] [12] [13]. Some of these studies examined the impact of differences between verbal processing and image processing on emotional states, and the results indicated that image processing has a stronger impact on emotions than does verbal processing [11] [12]. Hence, interventions asking clients to imagine positive future situations in their own lives using SFA questions, such as the miracle question or time machine question, are expected to not only provide clues for problem solving and building a better future, but also create a pleasant emotional or behavioral state for clients. The induction of positive emotions by positive future imagery prevents the worsening of negative emotions such as depression and anxiety [14], thereby having a direct preventive effect on emotional disorders.

In this study, we focused on the difference between image processing and verbal processing in information processing. Therefore, the purpose of this study was to examine the influence of differences in information processing on emotional states before and after interventions involving instructions on one's positive future using the question of SFA.

By studying the difference between image processing and verbal processing on emotional state in this study, it is possible to provide empirical knowledge about the effects of SFA interventions using image guidance. In addition, the findings obtained in this study will be useful in clarifying how the questioning technique developed from the theory of SFA changes the psychological state of the client.

As image processing has a stronger influence on emotional state than verbal processing of the same positive events [13] [15], we hypothesized that the emotional changes before and after the intervention will be greater in the imagery group than in the language description group, even when the same instructions for the SFA question are used. In addition, we predicted that the emotional state of the imagery group will change more positively than that of the language description group.

\section{Method}

\subsection{Participants}

The participants were 270 students (130 male, 140 female) from two universities 
in the Tohoku area of Japan. Their ages ranged from 18 to 26 years $(M=19.09$, $\mathrm{SD}=0.98)$. The participants were randomly assigned to either the imagery group or the language description group. Finally, there were 128 participants in the imagery group (61 male, 67 female) and 142 participants in the language description group (69 male, 73 female).

\subsection{Measures}

We used the Japanese version of the Positive and Negative Affect Schedule (PANAS), which was translated from the original version [16] by Sato and Yasuda [17]. The Japanese version consists of positive and negative affect scales (eight items each) to assess the present state of positive and negative affect in the participants. The positive affect scale includes items such as feeling proud and excited, whereas the negative affect scale includes items such as feeling afraid and nervous. The Japanese version demonstrated high reliability ( $\alpha=0.83$ for the positive affect scale and 0.82 for the negative affect scale) and validity, as assessed by Sato and Yasuda [17]. Its scoring is done along a six-point scale $(1=$ not at all to $6=$ very much).

The participants' subjective experience of the intervention was evaluated by asking the participants three questions at the end of the examination. These questions assessed emotional impression (What was the emotional impression of what you imagined (described in the worksheet) during the intervention task?), use of verbal and image processing (How much of the verbal or imagery processes did you use when performing the intervention task?), and task difficulty (How difficult was the intervention task?). The question on emotional impression used a six-point scale, ranging from 1 (extremely negative) to 6 (extremely positive), while the questions on use of verbal and imagery processes and task difficulty were rated on five-point scales, ranging from 1 (only verbal/extremely difficult) to 5 (only imagery/extremely easy).

\subsection{Intervention Task}

We adopted the time machine question [18] in the SFA to ask the participants about positive future self-images. It is employed in the SFA to help clients create a better future situation [19]. In the intervention of the imagery group, we used the instructions on the time machine question provided by Kawahara and Sasaki [19] to guide the clients in imagining their positive future. In contrast, in the language description group, we prepared a worksheet with reference to the instructions of Kawahara and Sasaki [19] to guide the clients in describing their future situation.

\subsection{Procedure}

The experiment was conducted in a small group of 20 - 80 participants during a psychology lecture class.

The experiments in this study followed the ethical code prescribed by the 
Japanese Psychology Association. The participants were informed by the experimenter about the purpose of the research, and assured of their right to privacy and voluntary participation in experiments. Only those participants who provided written informed consent were involved in the experiments.

The participants in the imagery group completed imagery practice using the instructions on cutting a lemon by Holmes et al. [13]. Those in the language description group practiced describing the visual, olfactory, and tactile experience of cutting a lemon in a worksheet.

After the practice, the participants in both groups completed the Japanese version of the PANAS before the intervention.

In the intervention task of the language description group, the experimenter first distributed the worksheets to the participants. Then, the experimenter asked the participants, "What will you be doing after 10 years?" and instructed them to describe the answer as a positive future situation in the worksheet. Following the instructions of the experimenter, the participants described their future situation in as much detail as possible with regard to each of the questions provided in the worksheet. The experimenter read each of these questions (i.e., the season, place, and surroundings of the positive future situation) from the worksheet while confirming that the participants answered the question.

In the intervention task of the imagery group, the experimenter first asked the participants, "What are you doing 10 years from now?" and then asked them to close their eyes. The participants were instructed to imagine as clearly as possible their future 10 years from the present time. Furthermore, the experimenter asked the participants about the season, place, and surroundings of their imagined future at intervals of about one minute. Finally, the experimenter instructed the participants to concentrate on their imagery.

After completing the intervention task, all participants completed the Japanese version of the PANAS after the intervention, and the three questions on their subjective experience of the intervention.

\subsection{Statistical Analyses}

Descriptive statistics were indicated through means and standard deviations. We performed t-tests to assess differences between the groups on the positive and negative affect scale scores of the Japanese version of the PANAS and the questions on subjective experience. Paired-samples t-tests were performed to assess change in PANAS scores for both groups before and after intervention. ANOVA was carried out to investigate changes in affect by the intervention task. All analyses were carried out using R version 3.1 [20].

\section{Results}

\subsection{Baseline Measures}

We performed a t-test on the participants' pre-intervention scores on the two scales of the Japanese version of the PANAS to assess differences in the affect le- 
vels of participants in the imagery and language description groups. No significant differences were found in the scores of the two groups (positive affect scale: $\mathrm{t}(268)=0.61, p=0.78$, negative affect scale: $\mathrm{t}(268)=1.08, p=0.28)$.

\subsection{Change in Affect by the Intervention Task}

First, to evaluate the hypothesis that mental imagery has a greater influence on emotion than language description, the participants' scores on the two scales of the Japanese version of the PANAS were analyzed using a mixed ANOVA, with time (before intervention, after intervention) as a within-subjects factor and intervention (imagery, language description) as a between-subjects factor. The analysis of the scores on the positive affect scale revealed main effects for time ( $F$ $[1,268]=31.74, p<0.001)$ and intervention $(F[1,268]=4.36, p<0.05)$. Additionally, a significant interaction was observed between time and intervention ( $F$ $[1,268]=7.40, p<0.01)$. Since the interaction between time and intervention was significant, we conducted a simple main effects test, in which the scores after intervention of the imagery group were significantly higher than those of the language description group $(F[1,268]=34.88, p<0.001)$. Similarly, the analysis of the scores on the negative affect scale revealed main effects for time $(F[1,268]$ $=31.40, p<0.001)$, and an interaction between time and intervention $(F[1,268]$ $=5.17, p<0.05)$, but no main effect for intervention. As the interaction between time and intervention was significant, we conducted a simple main effects test, in which the scores after intervention of the imagery group were significantly lower than those of the language description group $(F[1,268]=31.88, p<$ $0.001)$. Means and standard deviations for the Japanese version of PANAS for each intervention condition are shown in Table 1.

Second, we computed a variation of the PANAS scores on each scale for both intervention groups by referring to previous studies [3] [15] that calculated the extent of change in PANAS scores before and after the interventions. We then conducted a paired samples t-test to assess whether there were differences in the variation of the scores between the imagery and language description groups in each PANAS scale. As expected, the amount of change in the scores in the imagery group was greater than the change in the language description group in

Table 1. Means and standard deviations for the Japanese version of PANAS for each intervention condition $(\mathrm{n}=270)$.

\begin{tabular}{ccccc}
\hline & \multicolumn{2}{c}{ Imagery $(\mathrm{n}=128)$} & \multicolumn{2}{c}{ Language description $(\mathrm{n}=142)$} \\
\cline { 2 - 5 } & $\mathbf{M}$ & $\mathrm{SD}$ & $\mathbf{M}$ & $\mathrm{SD}$ \\
\hline Positive affect & & & & \\
Before & 21.43 & 6.92 & 20.90 & 7.09 \\
After & 25.16 & 8.83 & 22.21 & 7.92 \\
Negative affect & & & & \\
Before & 18.73 & 7.82 & 17.68 & 8.10 \\
After & 15.59 & 6.71 & 16.35 & 6.95 \\
\hline
\end{tabular}


both the positive and negative affect scales (positive affect scale: $\mathrm{t}(268)=2.72, p$ $<0.001$, negative affect scale: $\mathrm{t}(268)=-2.27, p<0.05)$. The variations of scores in the intervention groups for each PANAS scale are shown in Figure 1.

\subsection{Subjective Experience Checks}

To evaluate the participants' subjective experience during the intervention, we calculated the mean scores for emotional impression, imagery vividness, and intervention task difficulty for both groups. We then compared the scores of three questions between the imagery and language description groups using t-tests. The results indicated significant differences in the mean scores between the imagery and language description groups for all questions (emotional impression: $\mathrm{t}$ $(268)=2.03, p<0.05$; use of verbal and imagery processes: $\mathrm{t}(268)=10.99, p<$ 0.01 ; task difficulty: $\mathrm{t}(268)=-2.41, p<0.05)$. The scores of the imagery group were significantly higher than those of the language description group on the questions of emotional impression, and use of verbal and imagery processes. On the other hand, the score of the language description group was significantly higher than that of the imagery group on task difficulty. Means and standard deviations for the questions assessing subjective experience in both groups are shown in Table 2.

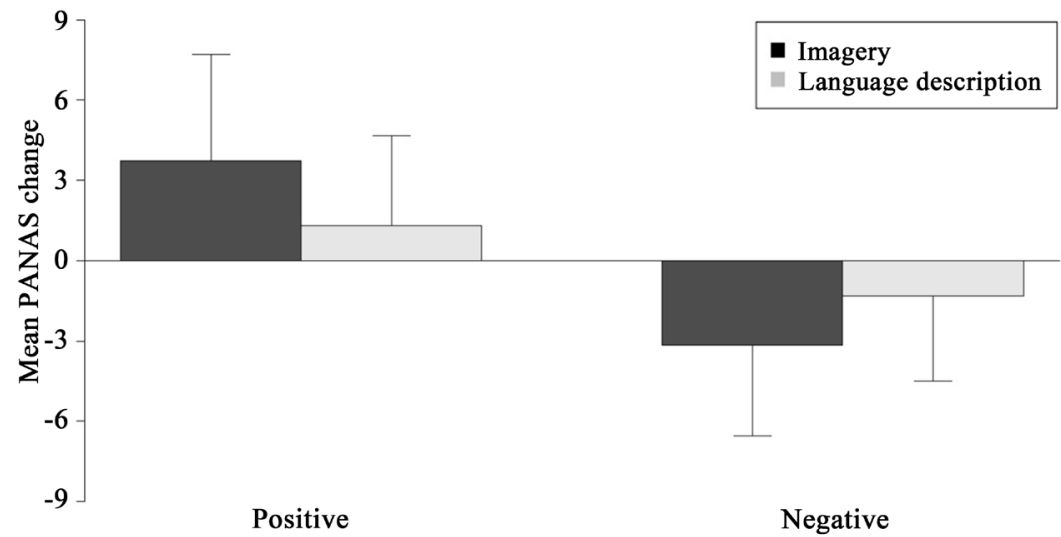

Figure 1. Change in PANAS scores before and after intervention in the imagery and language description conditions. Error bars show one standard deviation of the mean $(\mathrm{n}=$ 270).

Table 2. Means and standard deviations for subjective questions on the task for the imagery and language description groups $(\mathrm{n}=270)$.

\begin{tabular}{|c|c|c|c|c|c|}
\hline \multirow{2}{*}{ Aspect of rating } & \multicolumn{2}{|c|}{ Imagery $(n=128)$} & \multicolumn{2}{|c|}{$\begin{array}{l}\text { Language description } \\
\qquad(\mathrm{n}=142)\end{array}$} & \multirow[t]{2}{*}{$\mathrm{t}$} \\
\hline & $\mathbf{M}$ & $\mathrm{SD}$ & $\mathbf{M}$ & SD & \\
\hline Emotional impression & 5.34 & 1.49 & 4.96 & 1.50 & $2.03^{*}$ \\
\hline Use of verbal and imagery processes & 3.96 & 1.05 & 2.69 & 0.82 & $10.99^{* *}$ \\
\hline Task difficulty & 2.93 & 1.24 & 3.30 & 1.31 & $-2.41^{\star}$ \\
\hline
\end{tabular}




\section{Discussion}

In this study, we aimed to examine the influence of differences in information processing on emotional states before and after interventions involving instruction on describing one's positive future (verbal processing) or imagining it (image processing). This was done using the time machine question of the SFA. To achieve this purpose, we classified the participants into the language description group, which was instructed to use language to describe a positive future state in a worksheet, or the imagery group, which was instructed to imagine a positive future. We then compared the effects of the two intervention tasks on the emotional state of the two groups. We hypothesized that emotional changes in the imagery group would be greater than the changes in the language description group. The results indicated a significant difference in the amount of change in the positive and negative affect scores between the imagery and language description groups. For both scales, the change in scores in the imagery group was larger than that in the language description group. The evaluation of the subjective experience about information processing used during the intervention task showed that the participants in the imagery group used more imagery processing, and the participants in the language description group used more verbal processing during the intervention task, respectively. These results show that positive and negative affect changes, before and after the intervention, were greater for the imagery group than for the language description group, which is consistent with the findings of previous studies [13] [15], that image processing has a stronger influence on emotions than verbal processing. The visual information used in image processing can access the emotional system more directly than verbal information, because the emotional cues of the information can be used more quickly and easily [13]. Therefore, we speculate that imagery related to a positive future in the imagery group prompted the activation of the positive emotional system of the participants in this group, resulting in a greater change in emotional state, as compared to the language description group.

In addition, the imagery group showed positive improvements in emotional state after the intervention. This is in accordance with Holmes, Geddes, Colom, and Goodwin's [21] emotional amplifier model which states that the imagery of events acts as an amplifier that increases emotions. Considering this model, it could be that the participants in the imagery group may have amplified their positive emotions by imagining a positive future situation, resulting in a more positive improvement in their emotional state. This amplifying effect of positive imagery on emotional states may be also reflected in the result that the score on the emotional impression of the intervention task in the imagery group was higher than the language description group's score on the same.

Although participants in the imagery group showed greater emotional changes than the ones in the language description group, it is also possible that this difference was caused by the fact that the intervention tasks conducted with the language processing group were more difficult than those conducted with 
the imagery group. However, the result of the subjective experience about the difficult on the intervention task showed that the participants in the language description group rated the intervention task as easier than the participants in the imagery group. Therefore, it seems that the stronger effect on emotional state in the imagery group than in the language description group is not due to the difficulty of the intervention task. Considering these discussions, we concluded that the hypothesis of this study was supported by the results obtained.

\section{Conclusion}

In this study, we found that image processing has a stronger effect on emotional states than verbal processing. In the field of cognitive therapy, mental imagery has been argued as an important factor directly related to the effect of the intervention [11] [22]. However, in SFA, the discussion of emotional states between the counselor and the client during the intervention focuses on the client's problems, because of which little attention has been given to emotional changes in the client brought about by the questions of the counselor in SFA [6] [23]. The results obtained in this study suggest that interventions involving image processing of one's positive future situations using the SFAs questions have a more direct impact on emotional states than interventions using verbal processing. Hence, our results suggest that mental imagery plays an important role in interventions carried out within the framework of the SFA.

\section{Clinical Implications}

Empirical research on mental imagery suggests that increases of positive emotions by mental imagery improve motivation for action [4]. Therefore, imaging one's positive future increases positive emotions, and may help clients modify their behavior to solve their problems. The results of this study on the relationship between mental imagery and emotion can help understand the changes in cognitions or behaviors that begin from the SFA questions.

\section{Limitations and Future Challenges}

The study's limitations must also be considered. First, the answers of the participants to the three questions on their subjective experiences suggested that the participants in the language description group might also have imagined their future intervention, although to a lesser extent than the imagery group. In this regard, Holmes and Mathews [12] indicated that even tasks involving instructions that do not allow participants to imagine the situation using verbal processing methods, such as language description and speech to perform the task, are not able to completely remove the unconscious imagery. Therefore, to assess the effect of mental imagery on emotions in more detail, it is necessary to first establish the group conducting the task in which the participants avoid imagining as much as possible, and then compare the effect on emotion with the imagery group. In addition, we were unable to determine the length for which 
the effect of the imaging remained on the participants' emotional state. Therefore, further experiments are needed to add follow-up conditions that measure the participants' emotional state at regular intervals. Resolving the problems and limitations of this study, and adding experimental data on the relationship between mental imagery and emotional experience can help in providing more robust empirical evidence regarding the efficacy of mental imagery in SFA.

\section{Conflicts of Interest}

The author declares no conflicts of interest regarding the publication of this paper.

\section{References}

[1] Kim, J.S. and Franklin, C. (2009) Solution-Focused Brief Therapy in Schools: A Review of the Outcome Literature. Children and Youth Services Review, 31, 464-470. https://doi.org/10.1016/j.childyouth.2008.10.002

[2] Kim, J.S. (2008) Examining the Effectiveness of Solution-Focused Brief Therapy: A Meta-Analysis. Research on Social Work Practice, 18, 107-116. https://doi.org/10.1177/1049731507307807

[3] Holmes, E.A. and Mathews, A. (2005) Mental Imagery and Emotion: A Special Relationship? Emotion, 5, 489-497. https://doi.org/10.1037/1528-3542.5.4.489

[4] Renner, F., Ji, J.L., Pictet, A., Holmes, E.A. and Blackwell, S.E. (2017) Effects of Engaging in Repeated Mental Imagery of Future Positive Events on Behavioural Activation in Individuals with Major Depressive Disorder. Cognitive Therapy and Research, 41, 369-380. https://doi.org/10.1007/s10608-016-9776-y

[5] Berg, I.K. and De Jong, P. (1996) Solution-Building Conversations: Co-Constructing a Sense of Competence with Clients. Families in Society, 77, 376-391. https://doi.org/10.1606/1044-3894.934

[6] Ng, K.M., Parikh, S. and Guo, L. (2012) Integrative Solution-Focused Brief Therapy with a Chinese Female College Student Dealing with Relationship Loss. International Journal for the Advancement of Counselling, 34, 211-230. https://doi.org/10.1007/s10447-012-9152-x

[7] Bell, R.J. and Thompson, C.L. (2007) Solution-Focused Guided Imagery for a Golfer Experiencing the Yips: A Case Study. Athletic Insight, 9, 52-66.

[8] Sklare, G.B., Sabella, R.A. and Petrosko, J.M. (2003) A Preliminary Study of the Effects of Group Solution-Focused Guided Imagery on Recurring Individual Problems. Journal for Specialists in Group Work, 28, 370-381. https://doi.org/10.1177/01933922030284009

[9] Bell, R.J., Skinner, C.H. and Fisher, L.A. (2009) Decreasing Putting Yips in Accomplished Golfers via Solution-Focused Guided Imagery: A Single-Subject Research Design. Journal of Applied Sport Psychology, 21, 1-14.

https://doi.org/10.1080/10413200802443776

[10] Bell, R.J., Skinner, C.H. and Halbrook, M.K. (2011) Solution-Focused Guided Imagery as an Intervention for Golfers with the Yips. Journal of Imagery Research in Sport and Physical Activity, 6, 1-16. https://doi.org/10.2202/1932-0191.1059

[11] Hackmann, A., Bennett-Levy, J. and Holmes, E.A. (2011) Oxford Guide to Imagery in Cognitive Therapy. Oxford University Press, Oxford. https://doi.org/10.1093/med:psych/9780199234028.001.0001 
[12] Holmes, E.A. and Mathews, A. (2010) Mental Imagery in Emotion and Emotional Disorders. Clinical Psychology Review, 30, 349-362. https://doi.org/10.1016/j.cpr.2010.01.001

[13] Holmes, E.A., Mathews, A., Dalgleish, T. and Mackintosh, B. (2006) Positive Interpretation Training: Effects of Mental Imagery versus Verbal Training on Positive Mood. Behavior Therapy, 37, 237-247. https://doi.org/10.1016/j.beth.2006.02.002

[14] Renner, F. and Holmes, E.A. (2018) Mental Imagery in Cognitive Therapy: Research and Examples of Imagery-Focused Emotion, Cognition, and Behavior Change. In: Leahy, R.L., Ed., Science and Practice in Cognitive Therapy: Foundations, Mechanisms, and Applications, The Guilford Press, New York, 142-158.

[15] Nelis, S., Vanbrabant, K., Holmes, E.A. and Raes, F. (2012) Greater Positive Affect Change after Mental Imagery than Verbal Thinking in a Student Sample. Journal of Experimental Psychopathology, 3, 178-188. https://doi.org/10.5127/jep.021111

[16] Watson, D., Clark, L.A. and Tellegen, A. (1988) Development and Validation of Brief Measures of Positive and Negative Affect: The PANAS Scales. Journal of Personality and Social Psychology, 54, 1063-1070. https://doi.org/10.1037/0022-3514.54.6.1063

[17] Sato, A. and Yasuda, A. (2001) Development of the Japanese Version of Positive and Negative Affect Schedule (PANAS) Scales. The Japanese Journal of Personality, 9, 138-139. https://doi.org/10.2132/jjpjspp.9.2_138

[18] Kurosawa, S. and Mori, T. (2002) Time Machine Question-One Technique in Intention Approach in the Future (Time Machine Question-Mirai-Shikou Approach Niokeru Ichi-Gihou). Japanese Journal of Brief Psychotherapy, 11, 27-37.

[19] Kawahara, M. and Sasaki, M. (2019) The Effect of Mental Imagery on Intervention Using the Time Machine Question: Examining Changes in Emotion and Differences in Mental Imagery among Individuals. Japanese Journal of Brief Psychotherapy, 27, 50-61. https://www.jstage.jst.go.jp/article/jabp/27/2/27_270202/_article/-char/ja/

[20] R Core Team (2014) R: A Language and Environment for Statistical Computing. R Foundation for Statistical Computing, Vienna. https://www.R-project.org

[21] Holmes, E.A., Geddes, J.R., Colom, F. and Goodwin, G.M. (2008) Mental Imagery as an Emotional Amplifier: Application to Bipolar Disorder. Behaviour Research and Therapy, 46, 1251-1258. https://doi.org/10.1016/j.brat.2008.09.005

[22] Holmes, E.A., Arntz, A. and Smucker, M.R. (2007) Imagery Rescripting in Cognitive Behaviour Therapy: Images, Treatment Techniques and Outcomes. Journal of Behavior Therapy and Experimental Psychiatry, 38, 297-305. https://doi.org/10.1016/j.jbtep.2007.10.007

[23] King, E. (1998) Role of Affect and Emotional Context in Solution-Focused Therapy. Journal of Systemic Therapies, 17, 51-64. https://doi.org/10.1521/jsyt.1998.17.2.51 